\title{
Totais decendiais de precipitação pluvial em Campinas, SP: persistência temporal, periodicidades e tendências climáticas
}

\author{
Ten-day rainfall amounts at Campinas, State of São Paulo, Brazil: temporal persistence, \\ periodicities, and climate trends
}

\author{
Gabriel Constantino Blain ${ }^{\mathrm{I}}$
}

\section{RESUMO}

\begin{abstract}
O objetivo do estudo foi descrever as componentes tendências, periodicidades e persistência temporal presentes nas séries decendiais de precipitação pluvial (PRE) na localidade de Campinas/SP, entre os anos de 1890 e 2009. A aplicação da função auto-correlação e do teste Run permitiu inferir a inexistência de significativas correlações seriais entre os totais decendiais de PRE. Análises espectrais conduzidas no domínio tempo-frequencia não possibilitaram a indicação de significativas periodicidades nos picos de variância do sinal temporal PRE. Com base em métodos não paramétricos e considerando as probabilidades máximas de ocorrência do erro tipo I, usualmente admitidas na literatura (10 e 5\%), não houve indicação de marcantes tendências climáticas nas séries analisadas. Contudo, observou-se concentração de casos de tendências de queda a partir do final da época seca regional (mês de agosto) até o início do mês de novembro (pertencente à estação chuvosa). Essa característica pode ser considerada um indício de mudança nos padrões do clima regional no sentido de atraso da retomada da estação chuvosa.
\end{abstract}

Palavras-chave: Mann-Kendall, ondaletas, função autocorrelação.

\section{ABSTRACT}

The aim of the study was to evaluate the presence of temporal persistence, periodicals components, and trends within the ten-day rainfall series (PRE), observed during 1890-2009 at the weather station of Campinas, State of São Paulo, Brazil. Based on the autocorrelation function and on the Run test it was observed no significant temporal persistence within the data sample. The spectral analysis carried on the time-frequency domain has shown no remarkable periodicity associated with the variance peaks in the temporal signal under evaluation. Based on non parametric methods and considering the significance levels commonly adopted in the scientific literature (10 and 5\%) it was detected no important climate trend within the PRE series. However, during the end of the dry season and the beginning of the rainy season it was observed an unexpected high number of decreasing (negative) trends cases that may be an indication of changes in the climatic conditions observed during 1890-2009. This last feature may reveal a delay in the resumption of the rainy season.

Key words: Mann-Kendall, wavelets, autocorrelation function.

\section{INTRODUÇÃO}

Alterações de ordem climática na variabilidade temporal da precipitação pluvial (PRE) são uma das maiores preocupações da agrometeorologia, devido à possibilidade de redução na disponibilidade hídrica às culturas. De acordo com BLAIN \& PIRES (2011), mudanças ou variações nos padrões climáticos podem reduzir as trocas de fluidos (tais como, solução de sais minerais e componentes orgânicos, oxigênio e gás carbônico) no sistema solo-planta-atmosfera devido à elevação da ocorrência de estresse hídrico.

Com início em 1890, a série de precipitação pluvial composta pelos totais mensais observados na estação meteorológica de Campinas/SP vem sendo utilizada em estudos voltados à detecção de tendências climáticas. Embora os estudos de BLAIN et al. (2007), BLAIN et al. (2009) e BLAIN (2009) indiquem que a principal característica da PRE mensal nessa localidade é

'Centro de Ecofisiologia e Biofísica, Área de Climatologia, Instituto Agronômico (IAC), Av. Barão de Itapura, CP 28, 13012-

970, Campinas, SP, Brasil. E-mail: gabriel@iac.sp.gov.br. Autor para correspondência 
a elevada variabilidade temporal dos dados, sob a qual meses extremamente úmidos podem preceder ou serem precedidos por meses extremamente secos, BLAIN (2010) ressalta, para Campinas, a existência de tendências de elevação nos totais mensais desse elemento meteorológico, entre 1948 e 2007, especialmente marcada no mês de maio. Essa indicação parece corroborar HAYLOCK et al. (2006), que, ao analisar séries de PRE da América do Sul (1960-2000), apontam alterações para condições mais úmidas na região em que Campinas está inserida. HAYLOCK et al. (2006) e BLAIN (2010) utilizaram, respectivamente, as estatísticas não paramétricas Kendall (KENDALL, 1938) e o teste de Mann-Kendall (MANN, 1945; KENDALL \& STUART, 1967) nas análises de tendências temporais.

Embora a relevância de trabalhos como os de BLAIN (2010), restritos à escala mensal, seja apoiada por afirmações como as de CARGNELUTTI FILHO et al. (2010) de que a precipitação pluvial é importante para o adequado planejamento das atividades agropecuárias, a análise de totais mensais de PRE permanece distante dos interesses agrometeorológicos, tendo em vista que períodos de, por exemplo, 10 ou 20 dias, em que não há observações de ocorrência de PRE, podem ter impactos significativos na produção agrícola, dependendo da época ou estádio fenológico de ocorrência.

Além de investigações relativas a tendências climáticas, análises da possível presença de significativas componentes periódicas também desempenham papel importante na caracterização da variabilidade temporal da PRE. Para este fim, autores como NAKKEN (1998), REBOITA et al. (2006), BLAIN (2009) e BLAIN \& KAYANO (2011) utilizam a forma de investigação espectral denominada de análise de ondaleta (AO). Conforme TORRENCE \& COMPO (1998), a AO é um método superior à análise de Fourier no estudo de séries temporais com presença de componentes não estacionárias. Em adição, após afirmar que a $\mathrm{AO}$ é importante para investigações relativas ao tema "mudança climática", NAKKEN (1998) indica mudança na variabilidade temporal da precipitação pluvial e do escorrimento superficial entre os anos de 1911 e 1996 na região de South Wales, Austrália. Segundo esse autor, foi detectada alteração na frequência dominante desses sinais temporais após 1950.

Dessa forma, a fim de caracterizar a variabilidade temporal da precipitação pluvial decendial na região de abrangência da estação meteorológica de Campinas, o objetivo do trabalho foi descrever, em base estatística, as componentes tendências, periodicidades e persistência temporal presentes nas séries temporais do referido elemento meteorológico entre os anos de 1890 e2009.

\section{MATERIAL E MÉTODOS}

Foram utilizados dados decendiais de totais de PRE pertencentes ao Instituto Agronômico (IAC/ SAA-SP; Campinas 1890 a 2009; 22 ${ }^{\circ} 54^{\prime}$ 'S; 4705'W; 669m). De acordo com a classificação de Köepen, o clima local é Cwa, subtropical, com estação seca no inverno.

Conforme descrito em PANOFSKY \& BRIER (1968), OMM (1975) e WILKS (2006), para variáveis contínuas, a persistência temporal é tipicamente caracterizada em termos de correlação serial ou autocorrelação. Dessa forma, o método de Wald-Wofowitz (Run), conforme descrito em MORETTIN \& TOLOI (2006), e a função auto-correlação (acf), descrita em WILKS (2006), foram utilizados na quantificação da persistência temporal (ou correlação serial) presente na série temporal de PRE. Os coeficientes da acf foram calculados até o deslocamento (lag) 36 correspondes ao período de um ano. Esse número de lags foi arbitrariamente escolhido, respeitando-se, entretanto, o limite N/4, em que $\mathrm{N}$ é o número de dados da série. A hipótese de nulidade (Ho) associada ao teste Run e a acf, que indica que a série sob investigação pode ser considerada livre de persistência temporal (inexistência de significativas correlações seriais), não será rejeitada para valores de significância $\mathrm{P}>0,05$. É oportuno ressaltar que processos físicos que dão origem a séries temporais constituídas de dados independentes (ou seja, séries livres de persistência temporal) são denominados de ruído branco.

A periodicidade dos picos de variância presentes no sinal temporal foi investigada utilizando-se aAO conforme descrita em TORRENCE \& COMPO(1998) e REBOITA et al. (2006), com base na ondaleta mãe "Morlet". Esse último tipo de ondaleta foi utilizado por NAKKEN (1998), REBOITA et al. (2006), BLAIN (2009)e BLAIN \& KAYANO (2011) em demais estudos relativos ao sinal temporal da PRE. AAO foi conduzida utilizando o algoritmo elaborado por C. Torrence, disponível em http://paos.colorado.edu/research. O nível de significância, relativo ao espectro de fundo, adotado para condução da $\mathrm{AO}$ foi calculado segundo TORRENCE \& COMPO (1998) baseando-se no quantil 0,95 da distribuição qui-quadrado (2 graus de liberdade) e nos coeficientes da acf relativos aos dois primeiros lags. A AO foi aplicada aos resíduos decendiais da PRE, os quais foram obtidos subtraindo-se de cada valor de PRE sua respectiva mediana decendial. Com base nas nomenclaturas utilizadas emPANOFSKY \& BRIER(1968), TORRENCE \& COMPO(1998), GRINSTEDetal. (2004)e WILKS (2006), os picos de variância do sinal espectral também foram denominados de picos de potência ou picos de energia do espectro da ondaleta. 
O teste não paramétrico de Mann-Kendall (MK), quando comparado a outros métodos paramétricos, é bastante robusto quanto aos desvios da normalidade e à não-estacionaridade dos dados, justificando o fato do MK ser muito utilizado em estudos de tendências em séries temporais meteorológicas. Contudo, é necessário ressaltar que a presença de persistência temporal e/ou componentes sazonais afetam a sensibilidade desse teste de tendência (HAMED \& RAO, 1998 e BAYAZIT \& ONOZ, 2007). Com isso, a fim bem como lidar com dificuldades impostas pela presença de sazonalidades na série sob análise, HIRSCH et al. (1982) e HIRSCH \& SLACK (1984) propuseram o método denominado de Mann-Kendall Sazonal (SMK). Para LETTENMAIER et al. (1994), a vantagem da utilização do SMK, em relação ao método original de MK, relaciona-se ao fato de que alterações em estações em que são esperados baixos valores das variáveis sob investigação não são influenciadas por elevados valores oriundos de outras épocas. Para o caso de inexistência dessas componentes, as estatísticas MK e SMK tendem a convergir para um valor comum. O SMK foi utilizado a fim de verificar possíveis tendências, de elevação ou queda, presente nos dados. Valores positivos desse método não paramétrico indicam que a série sob análise apresenta tendência de elevação em seus dados. Valores negativos do SMK indicam tendência de queda. A significância associada à estatística SMK (valor P) é estimada com base na distribuição Gaussiana conforme descrito em HAMED \& RAO (1998) e BAYAZIT \& ONOZ (2007). YUE \& HASHINO (2003) afirmam que, embora o nível crítico usualmente associado à estatística de MannKendall seja probabilidade $\mathrm{P}=0,05$ de ocorrência do erro estatístico tipo I, séries livres de tendência exibirão valores deste teste próximos a zero associados, evidentemente, a valores $\mathrm{P} \geq 0,50$.

O teste não paramétrico de Pettitt (PETTITT, 1979), utilizado por VICTORIA et al. (1997)e MORTATTI et al. (2004) para indicar o ponto inicial de alteração em séries meteorológicas e hidrológicas, foi aplicado nas séries decendiais de PRE em que foram verificados valores do SMK com significância estatística $\mathrm{P}<0,20$.

\section{RESULTADOS E DISCUSÃO}

Após análise dos valores dos coeficientes da função auto-correlação $\left(\mathrm{r}_{\text {acf }}\right)$, bem como da significância associada aos mesmos, torna-se possível indicar que a série de PRE decendial na estação meteorológica de Campinas é livre de persistência temporal, uma vez que praticamente todos os $r_{\text {acf }}$ permanecem dentro do limite de ruído branco (Figura 1). Em outras palavras, essa inexistência de memória significativa na referida série permite inferir que a probabilidade de ocorrência de determinado total de PRE, observado no decêndio $X_{t}(t \in Z$; origem temporal), nao pode ser considerada significativamente condicionada pelo valor de PRE observado em $X_{t-1}$.

As características indicadas na figura 1 são corroboradas pelo teste Run (Tabela 1), tendo em vista que todos os 36 valores finais desta estatística permaneceram contidos no intervalo $-1,96 \leq \mathrm{Run} \leq 1,96$ $(\mathrm{P} \geq 0,05)$. Dessa forma, com base nos resultados da acf e do teste Run, a série de PRE da estação meteorológica de Campinas foi considerada livre de persistência temporal.

Analisando o sinal temporal da PRE apenas no domínio da frequência, verificam-se dois picos significativos de variância próximos às escalas dois e quatro anos (Figura $2 b$; potencia global da ondaleta). Conceitualmente, essa característica espectral indica significativa periodicidade nos picos de potência do sinal temporal da PRE nas referidas escalas. Contudo, estendendo essa análise para o espaço tempo-frequencia, observa-se que esses picos de variância concentram-se, fundamentalmente, entre os anos de 1920 e 1940 e entre

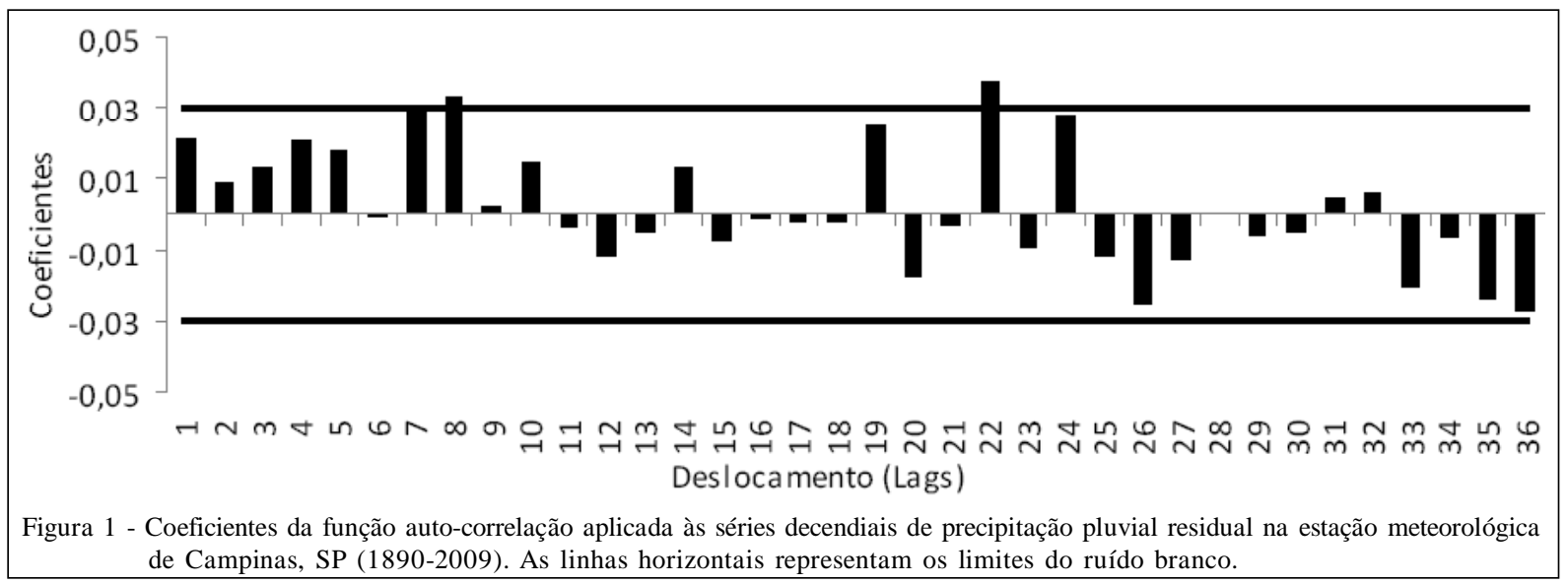

Ciência Rural, v.41, n.5, mai, 2011. 
Tabela 1 - Teste Run aplicado a séries decendiais (Dec) da estação meteorológica de Campinas, SP (1890-2009).

\begin{tabular}{llllll}
\hline Mês & Dec & Run & Mês & Dec & Run \\
\hline Janeiro & 1 & 0,20 & Julho & 19 & 0,12 \\
Janeiro & 2 & 0,69 & Julho & 20 & 0,20 \\
Janeiro & 3 & $-0,22$ & Julho & 21 & $-1,28$ \\
Fevereiro & 4 & $-0,02$ & Agosto & 22 & $-0,05$ \\
Fevereiro & 5 & 0,67 & Agosto & 23 & $-0,22$ \\
Fevereiro & 6 & 0,54 & Agosto & 24 & 0,97 \\
Março & 7 & 0,22 & Setembro & 25 & $-1,87$ \\
Março & 8 & 0,34 & Setembro & 26 & 0,06 \\
Março & 9 & 1,66 & Setembro & 27 & 0,26 \\
Abril & 10 & $-0,05$ & Outubro & 28 & 0,83 \\
Abril & 11 & 1,70 & Outubro & 29 & $-0,09$ \\
Abril & 12 & $-0,30$ & Outubro & 30 & $-1,15$ \\
Maio & 13 & 0,06 & Novembro & 31 & $-0,47$ \\
Maio & 14 & 1,86 & Novembro & 32 & 0,44 \\
Maio & 15 & $-0,79$ & Novembro & 33 & 0,34 \\
Junho & 16 & $-0,17$ & Dezembro & 34 & $-0,59$ \\
Junho & 17 & 1,90 & Dezembro & 35 & $-0,03$ \\
Junho & 18 & $-0,17$ & Dezembro & 36 & 0,39 \\
\hline
\end{tabular}

os inícios das décadas de 1960 e 1990, permanecendo praticamente ausentes nos demais anos da série (Figura 2a; potencia local da ondaleta). Nas escalas 2-8 anos (Figura 2c), nota-se queda da potência da ondaleta, ocorrida entre o final da década de 1940 e início da década de 1960. Salienta-se também que a analise das Figuras $2 \mathrm{a}$ e $2 \mathrm{c}$ evidencia declínio da energia espectral no sinal temporal da PRE após 1990. Na figura 2a, é possível identificar um pico significativo de variância, na escala 5-8 anos, fundamentalmente localizado no início do século XX. Para essa mesma escala, é também possível observar acúmulo de energia entre o final da década de 1970 e início de 1980.

Por último, e de forma geral, infere-se que a natureza esparsa dos picos de energia ilustrados na figura $2 \mathrm{a}$ não permite a indicação de alteração na frequência dominante no sinal da PRE ao longo dos 120 anos analisados. Nesse aspecto, a análise da figura 2 parece corroborar BLAIN (2009), ilustrando que a principal característica da precipitação pluvial observada em Campinas é a elevada variabilidade presente no sinal temporal desse elemento meteorológico.

Quando analisados apenas sob o ponto de vista numérico/estatístico, os resultados da aplicação do SMK corroboram a hipótese de inexistência de marcantes alterações de ordem climática na variabilidade temporal da PRE decendial em Campinas (Tabela 2). Valores deste teste de tendência associados a significâncias $\mathrm{P} \leq 0,10$ são observadas em apenas cinco dos 36 decêndios, sendo que somente no segundo decêndio de Julho o limite $\mathrm{P}=0,05$ foi ultrapassado.
Contudo, segundo RADZIEJEWSKI \& KUNDZEWICZ (2004) a presença de componentes relacionadas à mudança nos padrões climáticos em uma série temporal nem sempre é detectada com satisfatória significância por métodos estatísticos. Para esses autores, a não indicação da presença de tendências na série sob análise nem sempre pode ser interpretada como ausência de toda e qualquer alteração de ordem climática. Essa última característica, inerente à investigação de mudanças climáticas em séries temporais, foi também descrita em OMM (1975). Segundo esse documento, embora a queda da temperatura média de inverno observada nas planícies da Inglaterra entre 1910 e 1975 ainda não tivesse atingido um nível considerável de significância, o aumento da frequência de nevascas, associado a essa queda não significativa da temperatura, havia ultrapassado elevados níveis de significância. BLAIN \& PIRES (2011) corroboram as indicações da OMM (1975) e de RADZIEJEWSKI \& KUNDZEWICZ(2004) ao afirmarem que alterações pouco significativas em séries de evapotranspiração potencial e precipitação pluvial (associadas, por exemplo, a probabilidades do erro estatístico tipo I $\mathrm{P} \geq 0,10$ ) podem resultar em elevações ou queda (com significâncias $\mathrm{P} \leq 0,05$ ) em parâmetros relativos à demanda hídrica das culturas como, por exemplo, a evapotranspiração real.

Dessa forma, sob uma análise qualitativa, torna-se oportuno verificar que entre o auge da estação seca (Agosto) e o primeiro decêndio do mês de Novembro (estação chuvosa regional), há marcante predomínio de casos de tendências de queda nos totais decendiais de PRE. Oito dos dez decêndios que compõem este período apresentam valores negativos do SMK (decêndios 22, 23, 24, 25, 27, 29, 30 e 31; Tabela 2). Independente da significância associada ao valor final do teste, essa característica pode ser considerada distante do que seria esperado em séries temporais (perfeitamente) livres de tendência, em que casos positivos e negativos do SMK tendem a distribuir-se de forma uniforme ao longo dos decêndios. Futuros estudos poderão, portanto, verificar a possível influência desse predomínio de tendências negativas nos totais de PRE em atividades agrícolas, como início da semeadura/plantio. De forma geral, os valores da estatística SMK associados à significância $\mathrm{P} \leq 0,20$ tendem a concentrar-se fora dos meses chuvosos da região. Essa última condiçãoé especialmente notável nos casos de tendências positivas (decêndios 9, 11, 13 e 21; Tabela 2). Tendências de queda, associadas à $P \leq 0,20$, são observadas tanto em decêndios pertencentes à estação chuvosa (6 e 7) quanto em épocas mais secas (decêndios 23 e 27; Tabela 2). Salienta-se também que o teste de Pettitt não foi capaz de indicar, de forma concomitante, a data inicial das tendências descritas na tabela 2. 


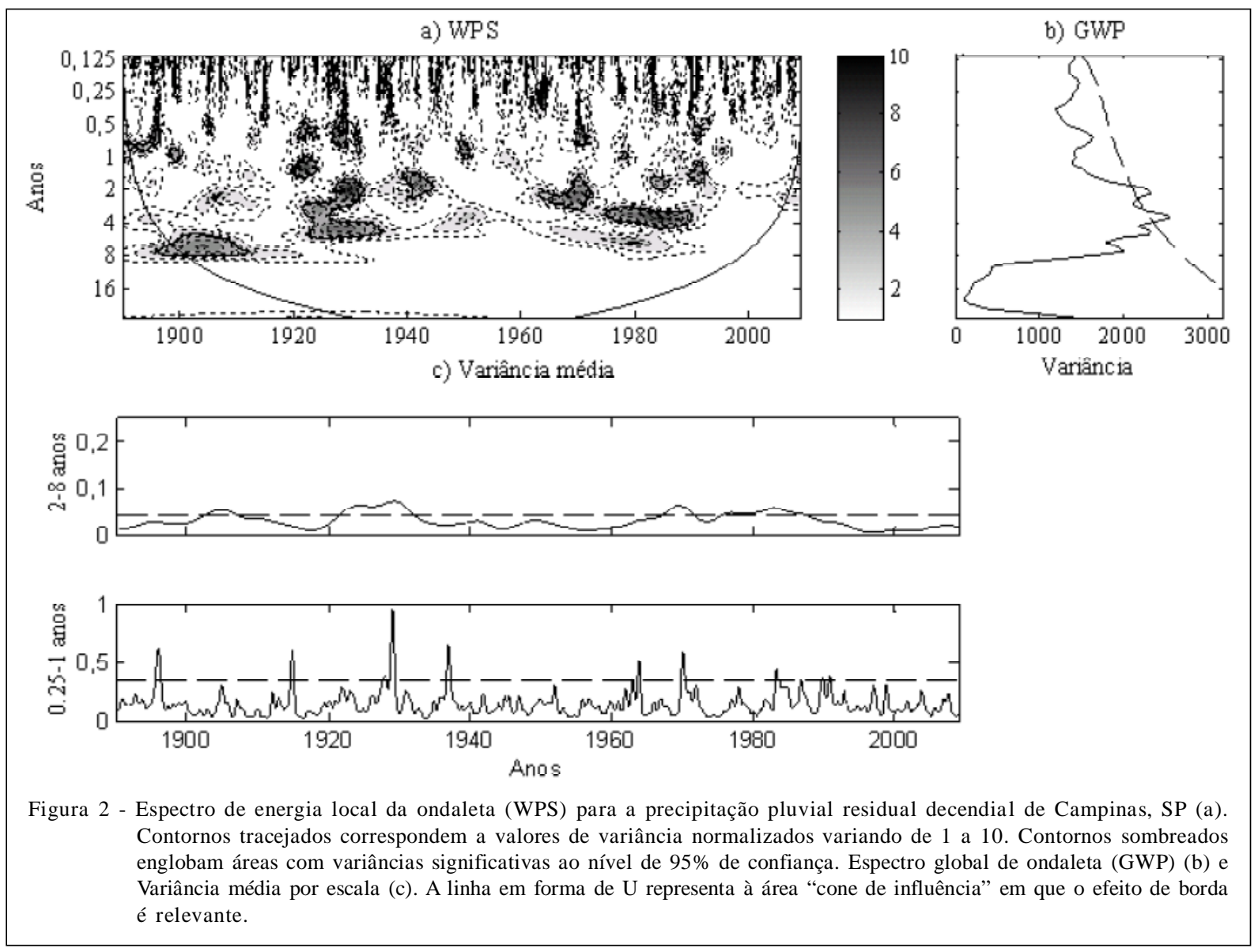

Tabela 2 - Teste sasonal de Mann-Kendall (SMK) relativo às séries decendiais (Dec) em Campinas, SP (1890-2009).

\begin{tabular}{llllllll}
\hline Mês & Dec & SMK & $\mathrm{p}$ & Mês & Dec & SMK & $\mathrm{p}$ \\
\hline Janeiro & 1 & 0,73 & 0,46 & Julho & 19 & $-1,08$ & 0,28 \\
Janeiro & 2 & $-0,21$ & 0,83 & Julho & 20 & 0,94 & 0,35 \\
Janeiro & 3 & 0,19 & 0,85 & Julho & 21 & 2,22 & $0.03(1964)$ \\
Fevereiro & 4 & $-0,68$ & 0,49 & Agosto & 22 & $-1,07$ & 0,29 \\
Fevereiro & 5 & 0,40 & 0,69 & Agosto & 23 & $-1,83$ & $0.07(1937)$ \\
Fevereiro & 6 & $-1,39$ & $0.17(1967)$ & Agosto & 24 & $-0,26$ & 0,79 \\
Março & 7 & $-1,59$ & $0.11(1946)$ & Setembro & 25 & $-0,43$ & 0,67 \\
Março & 8 & $-0,37$ & 0,71 & Setembro & 26 & 0,43 & 0,67 \\
Março & 9 & 1,64 & $0.10(1952)$ & Setembro & 27 & $-1,55$ & $0.12(1924)$ \\
Abril & 10 & 0,20 & 0,84 & Outubro & 28 & 1,19 & 0,23 \\
Abril & 11 & 1,86 & $0.06(1973)$ & Outubro & 29 & $-0,70$ & 0,49 \\
Abril & 12 & 0,10 & 0,92 & Outubro & 30 & $-0,88$ & 0,38 \\
Maio & 13 & 1,69 & $0.09(1970)$ & Novembro & 31 & $-1,11$ & 0,27 \\
Maio & 14 & $-0,02$ & 0,99 & Novembro & 32 & 0,22 & 0,83 \\
Maio & 15 & 0,98 & 0,33 & Novembro & 33 & $-0,37$ & 0,71 \\
Junho & 16 & $-0,62$ & 0,53 & Dezembro & 34 & $-0,08$ & 0,94 \\
Junho & 17 & $-0,45$ & 0,65 & Dezembro & 35 & 0,64 & 0,52 \\
Junho & 18 & $-0,41$ & 0,68 & Dezembro & 36 & $-0,31$ & 0,76 \\
\hline
\end{tabular}




\section{CONCLUSÃO}

A série de precipitação pluvial decendial de Campinas não apresenta significativa persistência temporal.

Com base apenas no domínio da frequência, observam-se dois picos de variância com periodicidades iguais a dois e quatro anos. Contudo, não é possível indicar a existência de marcadas periodicidades no sinal da variável sob estudo. Essa conclusão deve-se ao fato de que, sob o domínio tempo-frequência, observam-se longos períodos em que nenhum pico significativo de variância é verificado.

Há concentração de casos de tendências de queda nos totais decendiais de PRE entre o final da época seca regional (mês de agosto) e o início do mês de novembro (este último pertencente à estação chuvosa). Essa característica indica mudança nos padrões do clima regional no sentido de atraso da retomada da estação chuvosa.

\section{REFERÊNCIAS}

BAYAZIT, M.; ONOZ, B. To prewhiten or not prewhiten in trend analysis? Hydrologic Science Journal, v.52, p.611624, 2007. Disponível em: <http://www.informaworld.com/ $\mathrm{smpp} /$ content $\sim \mathrm{db}=\mathrm{all}$ ? content $=10.1623 / \mathrm{hysj} .52 .4 .611>$. Acesso em: 20 jan. 2011. doi: 10.1623/hysj.52.4.611.

BLAIN, G.C. et al. Distribuição temporal da precipitação pluvial mensal observada no Posto Meteorológico do Instituto Agronômico, em Campinas, SP. Bragantia, v.66, p.347355, 2007. Disponível em: <http://www.scielo.br/ scielo.php? script $=$ sci_arttext $\&$ pid $=$ S0006870520070002000 19\&lng=en\&nrm=iso $>$. Acesso em: 20 jan. 2011. doi: $10.1590 /$ S0006 87052007000200019 .

BLAIN, G.C. Considerações estatísticas relativas à oito séries de precipitação pluvial da Secretaria de Agricultura e Abastecimento do Estado de São Paulo. Revista Brasileira de Meteorologia, v.24, p.12-23, 2009. Disponível em: <http://www.scielo.br/ scielo.php? script =sci_arttext\&pid=S0102$77862009000100002 \& \operatorname{lng}=e n \& n r m=i s o>$. Acesso em: 21 jan. 2011. doi: 10.1590/S0102-77862009000100002.

BLAIN, G.C. et al. Variabilidade amostral das séries mensais de precipitação pluvial em duas regiões do Brasil: Pelotas-RS e Campinas-SP. Revista Brasileira de Meteorologia, v.24, p.1-11, 2009. Disponível em: <http://www.scielo.br/ scielo.php?script =sci_art text \& pid=S0102$77862009000100001 \& \operatorname{lng}=\mathrm{en} \& \mathrm{nrm}=\mathrm{iso}>$. Acesso em: 20 dez. 2010. doi: 10.1590/S0102-77862009000100001.

BLAIN, G.C. Detecção de tendências monótonas em séries mensais de precipitação pluvial do Estado de São Paulo. Bragantia, v.69, p.1027-1033, 2010. Disponível em: <http://www.scielo.br/ scielo.php?script $=$ sci_arttext\&pid $=$ S000687052010000400031\& $\operatorname{lng}=\mathrm{en} \& \mathrm{nrm}=\mathrm{iso}>$. Acesso em: 03 mar. 2011. doi: 10.1590/ S0006-87052010000400031.

BLAIN, G.C; PIRES, R.C.M. Variabilidade temporal da evapotranspiração real e da razão entre evapotranspiração real e potencial em Campinas, Estado de São Paulo. Bragantia, v.70, 2011 (prelo)

BLAIN, G.C; KAYAMO, M.T. 118 anos de dados mensais do índice padronizado de precipitação: série meteorológica de Campinas, Estado de São Paulo. Revista Brasileira de Meteorologia, v.24, 2011 (no prelo)

CARGNELUTTI FILHO, A. et al. Dimensionamento da amostra para a estimação da média de precipitação pluvial mensal em diferentes locais do Estado do Rio Grande do Sul. Ciência Rural, v.40, p.12-19, 2010. Disponível em: <http:// www.scielo.br/scielo.php? script $=$ sci_arttext\&pid $=$ S0 103 $84782010000100003 \& \operatorname{lng}=e n \& n r m=i s o>$. Acesso em: 20 nov. 2010. doi: $10.1590 / \mathrm{S} 0103-84782009005000226$.

GRINSTED, A. et al. Application of the cross wavelet transform and wavelet coherence to geophysical time series. Nonlinear processes in geophysics, v.11, p.561-566, 2004. Disponível em: <http://www.nonlin-processes-geophys.net/11/561/2004/ npg-11-561-2004.html>. Acesso em: 20 nov. 2010. doi: 10.5194/npg-11-561-2004

HAMED, K.H; RAO, A.R. A modified Mann-Kendall trend test for auto-correlated data. Journal of Hydrologic, v.204, p.182196, 1998. Disponível em: <http://linkinghub.elsevier.com/ retrieve/pii/S002216949700125X>. Acesso em: 20 nov. 2010. doi: 10.1016/S0022-1694(97)00125-X.

HAYLOCK, M.R. et al. Trends in total and extreme South American rainfall in 1960-2000 and links with sea surface temperature. Journal of Climate, v.19, p.1490-1512, 2006. Disponível em: <http://journals.ametsoc.org/doi/pdf/10.1175/ JCLI3695.1>. Acesso em: 20 nov. 2010. doi: 10.1175/ JCLI3695.1.

HIRSCH, R.M., et al. Techniques of trend analysis for monthly water quality data. Water Resources Research, v.18, p.107121, 1982. Disponível em: <http://www.agu.org/journals/ABS/ 1982/WR018i001p00107.shtml>. Acesso em: 20 nov. 2010. doi: 10.1029/WR018i001p00107.

HIRSCH, R.M.; SLACK, J.R. A nonparametric trend test for seasonal data with serial dependence. Water Resources Research, v.20, p.727-732, 1984. Disponível em: <http:// www.agu.org/journals/ABS/1984/WR020i006p00727.shtml>. Acesso em: 20 nov. 201. doi: 10.1029/WR020i006p00727.

KENDALL, M.G. A new measure of rank correlation. Biometrika, v.30, p.81-93, 1938 .

KENDALL, M.G.; STUART, A. The advanced theory of statistics. Londres: Charles Griffin \& Company, 1967. 690p.

LETTENMAIER, D.P. et al. Hydro-climatological trends in the continental United States, 1948-88. Journal of Climate, v.7, p.586-607, 1994. Disponível em: <http://journals.ametsoc.org/ toc/clim/7/4>. Acesso em: 20 nov. 2010. doi: 10.1175/15200442(1994)007<0586:HCTITC>2.0.CO;2.

MANN, H.B. Non-parametric tests against trend. Econometrica v.13, p.245-259, 1945. Disponível em: <http://www.jstor.org/ stable/1907187>. Acesso em: 20 nov. 2010.

MORETTIN, P.L.; TOLOI, C.M.C. Análises de séries temporais. 2.ed. São Paulo: Edgard Blucher, 2006. 531p. 
MORTATTI, J. et al. Hidrologias dos rios Tietê e Piracicaba: séries temporais de vazão e hidrogramas de cheia. Revista de Ciência e Tecnologia, v.12, p.55-67, 2004. Disponível em: <http://www.unimep.br/phpg/editora/revistaspdf/rct23art05.pdf>. Acesso em: 20 nov. 2010.

NAKKEN, M. Wavelet analysis of rainfall-runoff variability isolating climatic from anthropogenic patterns, Environmental Modelling \& Software, v.14, p.283-295, 1998. Disponível em: <http://linkinghub.elsevier.com/retrieve/ pii/S1364815298000802>. Acesso em: 20 nov. 2010. doi: 10.1016/S1364-8152(98)00080-2.

ORGANIZAÇÃO METEOROLÓGICA MUNDIAL. Climatic Change. Geneve: World Meteorological Organization, 1966. 79p. (OMM, 195; TP, 100; Technical note, 79).

PANOFSKY H.A.; BRIER G.W. Some applications of statistics to meteorology. Florida: University Park, 1968. $224 \mathrm{p}$.

PETTITT, A.N. A non-parametric approach to the changepoint problem. Journal of the Royal Statistical Society, v.28, p.126-135, 1979. Disponível em: <http://www.jstor.org/ pss/2346729>. Acesso em: 19 nov. 2010.

RADZIEJEWSKI, M.; KUNDZEWICZ, Z.W. Detectability of changes in hydrological records. Hydrological Sciences Journal, v.49, p.39-51, 2004. Disponível em: <http://dx.doi.org/ 10.1623/hysj.49.1.39.54002 >. Acesso em: 20 nov. 2010. doi: 10.1623/hysj.49.1.39.54002.

REBOITA, M.S. et al. Climate variability in Rio Grande, RS, Brazil: A quantitative analysis of contributions due to atmospheric systems. Revista Brasileira de Meteorologia, v.21, p.256270, 2006. Disponível em: <http://www.rbmet.org.br/port/revista/ revista_artigo.php?id_artigo=107>. Acesso em: 11 mar. 2011.

TORRENCE, C.; COMPO, G.P. A practical guide to wavelet Analysis, Bulletin of American Meteorological Society, v.79, p.61-78, 1998. Disponível em: <http://journals.ametsoc.org/toc/ bams/79/1>. Acesso em: 20 nov. 2010. doi: 10.1175/15200477(1998)079\%3C0061:APGTWA\%3E2.0.CO.

VICTORIA, R.L. et. al. Surface air temperature variations in the Amazon Region and Its borders during this century. Journal of Climate, v.11, p.1105-1110, 1997. Disponível em: <http:// journals.ametsoc.org/toc/clim/11/5>. Acesso em: 20 nov. 2008. doi: $10.1175 / 1520-0442(1998) 011<1105:$ SATVIT > 2.0.CO;2.

WILKS, D.S. Statistical methods in the atmospheric sciences. San Diego: Academic, 2006. 629p.

YUE, S.; HASHINO, M. temperature trends in Japan: 19001996. Theoretical and Applied Climatology, v.75, p.15-27, 2003. Disponível em: <http://www.springerlink.com/content/ j3pu5b5ptlemr03b/>. Acesso em: 20 nov. 2008. doi: 10.1007/ s00704-002-0717-11. 